\title{
Deep Alanine Scanning Reveals Potent Multi-alanine-substituted Protein-protein Interaction Inhibitors
}

Xiyun Ye, ${ }^{1 \dagger}$ Yen-Chun Lee, ${ }^{1 \dagger}$ Zachary P. Gates, ${ }^{1}$ Yingjie Ling, ${ }^{2}$ Jennifer C. Mortensen, ${ }^{2}$ Fan-Shen Yang, ${ }^{3}$ Yu-Shan Lin, ${ }^{2}$ Bradley L. Pentelute ${ }^{1,4,5,6 *}$

${ }^{1}$ Department of Chemistry, Massachusetts Institute of Technology, 77 Massachusetts Avenue, Cambridge, MA 02139, USA

2 Department of Chemistry, Tufts University, 62 Talbot Avenue, Medford, MA 02155, USA

${ }^{3}$ Department of Chemistry and Frontier Research Center on Fundamental and Applied

Sciences and Matters, National Tsing Hua University, 101, Sec. 2, Guang-Fu Road, Hsinchu 300, Taiwan

${ }^{4}$ The Koch Institute for Integrative Cancer Research, Massachusetts Institute of Technology, 500 Main Street, Cambridge, MA 02142, USA

5 Center for Environmental Health Sciences, Massachusetts Institute of Technology, 77 Massachusetts Avenue, Cambridge, MA 02139, USA

${ }^{6}$ Broad Institute of MIT and Harvard, 415 Main Street, Cambridge, MA 02142, USA

* Correspondence to: blp@mit.edu

Keywords: alanine scanning, synthetic peptide libraries, affinity selection, MDM2, peptide inhibitors.

\begin{abstract}
Establishing structure-activity relationships is crucial to understand and optimize the activity of peptide-based inhibitors of protein-protein interactions. Single alanine mutagenesis provides limited information toward this goal. To guide multiple simultaneous peptide modifications with retention of biological activity, we used synthetic combinatorial alanine-scanning libraries-in which each position was varied with either the wild type residue or alanine- with an affinity selection platform to study the mutational tolerance of protein-ligand interactions. Applying this platform to a peptide binder to the oncogenic protein MDM2, several multi-alanine-substituted analogs that retained low nanomolar affinity were discovered, including a 13-mer binder with seven alanine substitutions at non-hotspot positions. These binders served as templates for further modifications, generating cysteine-substituted, perfluoroaryl-stapled peptides with subnanomolar affinity and ten-fold improved proteolytic stability. The alanine substitution tolerances for peptide ligands of the $12 \mathrm{ca} 5$ antibody and 14-3-3 regulatory protein were also reported, demonstrating the general applicability of this new platform. We envision that deep combinatorial alanine scanning will be a powerful tool for structure-activity optimization of potential peptide therapeutics.
\end{abstract}

\section{INTRODUCTION}

Protein-protein interactions (PPIs) drive many aspects of biological function and are heavily involved in disease progression. The extensive $\left(1,000-5,000 \AA^{2}\right)$, shallow and flat PPI interface challenges the development of PPI modulators using small molecules ${ }^{1-3}$. Peptides, on the other hand, can mimic the native binding epitope to recognize the PPI interface with high binding affinity and specificity. Recent advances in affinity selections and biological display methods have accelerated the generation of peptide-based PPI 
inhibitors ${ }^{1,4}$. To improve biophysical and pharmacological properties, iterative optimization is necessary. This process involves various structural modifications, for example, side chain modifications or macrocyclization to develop better analogs ${ }^{5,6}$. Modifying peptides while maintaining their binding affinity is crucial for hit-to-lead drug development, thus calling for a deep understanding of structure-activity relationships $(\mathrm{SAR})^{7}$.

Alanine scanning informs SAR of peptides by systematically substituting each residue with alanine. This approach characterizes alanine tolerable residues and irreplaceable 'hotspot' residues essential for activity. Hotspots are identified by point alanine mutations that give rise to inactive mutants ${ }^{8}$. The alanine tolerable residues are often subjected to structure modifications without impacting the bioactivity. When multiple modifications happen simultaneously, non-additive combination effects emerge $^{9-13}$, leading to unforeseen boosts or disruptions in activity. Complementary to single-point alanine scanning, shotgun alanine scanning is widely employed in protein mutagenesis to interrogate the pairwise and higher order combination effect of multi-point mutations ${ }^{14-18}$. As an example, the phage-displayed $y$-receptor protein library was constructed by varying eleven residues to wild type (WT) or alanine ${ }^{19,20}$. The library was subjected to bioactivity assays to select for active strains, which presented a specific ratio of wild type to alanine at each residue. Pairwise analysis showed the frequency of most double alanine mutations followed a normal distribution when the two mutated residues were located in discontiguous regions ${ }^{14,21}$. In the case of peptides, however, an alanine mutation at one residue may affect the tolerance of an alanine mutation at a neighboring residue. An analysis of the combined effects of multi-site modifications is thus critical at revealing the comprehensive peptide SAR landscape.

We aimed to establish a platform to rapidly identify multiple sites in peptide-based lead compounds that tolerate modification while maintaining bioactivity. Toward this goal, we introduce here label-free combinatorial alanine library affinity selection (Figure 1A) based on our recent in-solution enrichment strategy for the discovery of PPI inhibitors ${ }^{22}$. Labelfree peptide libraries were synthesized by the split-and-pool solid phase peptide synthesis (SPPS) method. The peptide library was incubated with the target protein and subjected to high-performance size-exclusion chromatography (HPSEC) to separate bound and unbound ligands. The bound variants were decoded by liquid chromatography-tandem mass spectrometry (LC-MS/MS) ${ }^{23}$. This in-solution affinity selection is a powerful alternative to on-bead screening and provides fine control over the selection conditions.

The strategy was applied to the PMI peptide inhibitor of oncoprotein mouse double minute2 homolog (MDM2). The PMI sequence (TSFAEYWNLLSP-NH 2 ) with high affinity (dissociation constant, $K_{d}=7.7 \pm 4.5 \mathrm{nM}$ ) against MDM2 was discovered by phage display $^{24,25}$ (Figure 1B and C). MDM2 is an E3 ubiquitin ligase of the transcription factor p53 that responds to stress by promoting DNA repair, cell cycle arrest, senescence and apoptosis ${ }^{26}$. MDM2 recognizes the $\mathrm{N}$-terminal p53 transactive domain (p53 ${ }^{17-28}$, see Figure 1B for amino acid sequence) and promotes the ubiquitin-mediated p53 degradation. Disrupting p53-MDM2 binding is a strategy to restore p53 activity and promote apoptosis of cancer cells. For instance, a p53 epitope-derived hydrocarbon- 
stapled peptide (ATSP-7041) has entered phase II clinical trials ${ }^{27}$. Aided by the combinatorial alanine scanning technique developed here, we identified several PMI analogs with simultaneous multi-alanine substitutions that maintained high affinity for MDM2. In some cases, we found the multi-alanine PMI variants lead to active Cys substituted peptide macrocycles.

A) Split-and-pool library synthesis

(A) Alanine $\times$ WT amino acid

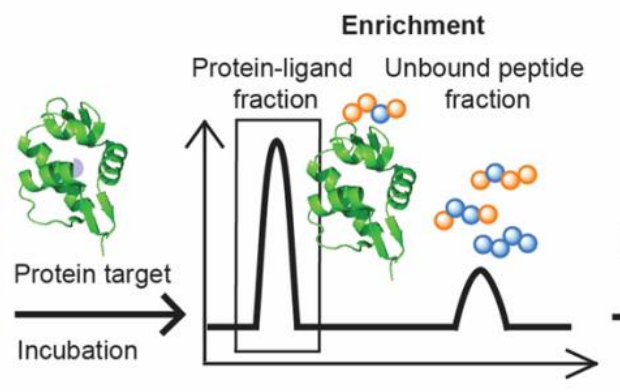

Enrichment

Identified sequences

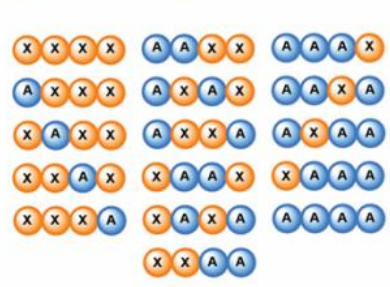

Combinatorial

WT/alanine library

HPSEC

affinity selection

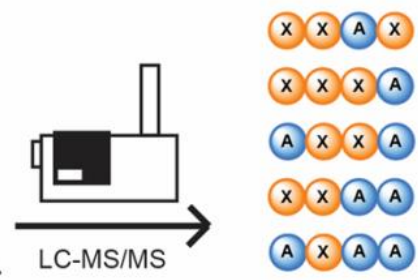

B)

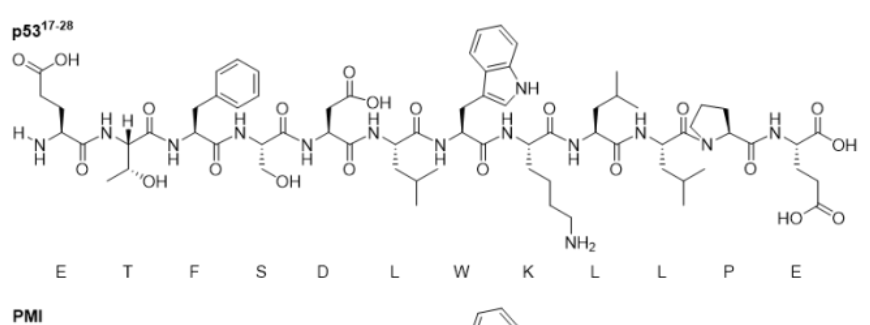

PMI

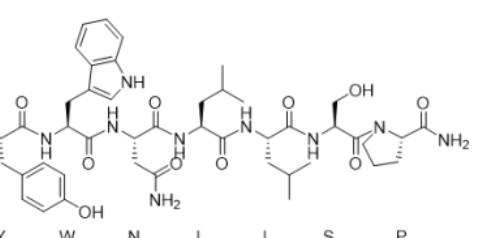

C)

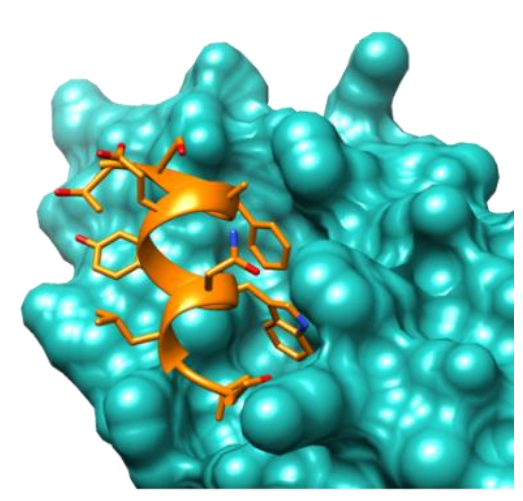

Figure 1. Combinatorial alanine scanning enables identification of alanine-containing peptide binders to proteins of interest. A) A chemically synthesized combinatorial alanine library was generated by split-and-pool solid phase peptide synthesis. The library was cleaved, deprotected, solid phase extracted, and incubated with protein targets. The peptide-protein complex eluate was separated from unbound peptides by size-exclusion chromatography. Bound peptide binders were dissociated from the protein and then sequenced by Q-TOF liquid chromatography-tandem mass spectrometry (LC-MS/MS). B) Chemical structure of p53 ${ }^{17-28}$ peptide (ETFSDLWKLLPE-OH) and PMI (TSFAEYWNLLSP-NH ${ }_{2}$ ). C) Crystal structure of the PMI-MDM2 complex (TSFAEYWNLLS-NH 2 , PDB entry 3LNZ) ${ }^{24}$.

\section{RESULTS}

The combinatorial alanine scanning platform was developed to identify alanine-containing peptide binders of the target protein (Figure 1). A PMI-derived combinatorial alanine library was prepared by split-and-pool SPPS. During synthesis, each position was evenly pooled to give either the wild-type amino acid or alanine, resulting in a library of 4,096 peptide variants. A $C$-terminal lysine is included to improve de novo LC-MS/MS sequencing. Prior to affinity selection, the library solution was incubated with MDM2 in Tris buffer $\mathrm{pH}=7.5$ to reach equilibrium. The peptide-protein complexes were enriched 
by HPSEC where the early protein fraction eluted. Bound peptides were dissociated from the protein, sequenced by LC-MS/MS and analyzed with the PEAKS software suite ${ }^{23}$. Identified sequences were filtered based on the library design ${ }^{23}$. To identify non-specific binders in the PMI-based library, we screened in parallel against the $12 \mathrm{ca} 5$ clone of antihemagglutinin antibody, for which no sequences were enriched.

\section{Single position SAR analysis}

Positional alanine substitution frequency (Figure 2A) can be used to differentiate binding hotspots from non-essential residues. Unique alanine mutant peptides were recovered from the affinity selection (Figure 2B). For each position, the alanine frequency was determined by dividing the number of alanine mutations to the total number of identified sequences, averaging by three replicates $\left(N_{\text {average }}=79\right)$. Four positions Phe3, Tyr6, Trp7 and Leu10 are of low alanine frequency (Ala\% <10\%), consistent with hotspots determined by point alanine mutagenesis ${ }^{25}$.

To provide quantitative data on residue-specific contributions to binding affinity, positional alanine frequencies from the combinatorial scanning were converted to changes in Gibbs free binding energy ( $\Delta \Delta G_{\text {scanning }}$ ). This calculation assumes that the ratio of WT to Ala for each position $\left(n_{\mathrm{WT}_{\mathrm{T}}} / n_{\mathrm{Ala}}\right)$ approximates the ratio of equilibrium association constants $K_{\mathrm{a}, \mathrm{WT}}$

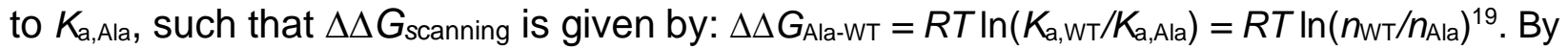
comparing the $\Delta \Delta G_{\text {Ala-WT }}$ values calculated from combinatorial scanning reported here ( $\Delta \Delta G_{\text {scanning }}$ ) to the $\Delta \Delta G_{\text {binding }}$ values previously measured by point alanine mutagenesis ${ }^{25}$, we found the two correlated linearly $\left(r^{2}=0.88\right.$, Figure S1). In particular, the $\Delta \Delta G_{\text {scanning }}$ values of the four hotspot residues (Phe3, Tyr6, Trp7 and Leu10) all $>1.0 \mathrm{kcal} / \mathrm{mol}$, consistent with the conventional definition of hotspot ${ }^{8}$. Therefore, the combinatorial alanine scanning informs on SAR at the single position level.

While $\Delta \Delta G_{s c a n n i n g}$ for each position derived from the combinatorial scanning correlated well with the previously reported $\Delta \Delta$ Gbinding values, the slope was found to be 0.36 . This significant deviation from 1.0 suggests a numerical discrepancy between the $\Delta \Delta G_{\text {scanning }}$ and $\Delta \Delta G_{\text {binding }}$ and indicates that combinatorial alanine scanning tends to underestimate the effect of individual alanine point mutations. This observation may result from the cooperative effect of alanine substitutions that compensate the energy cost of alanine substitutions. 
A) 1-D single position alanine substitution frequency

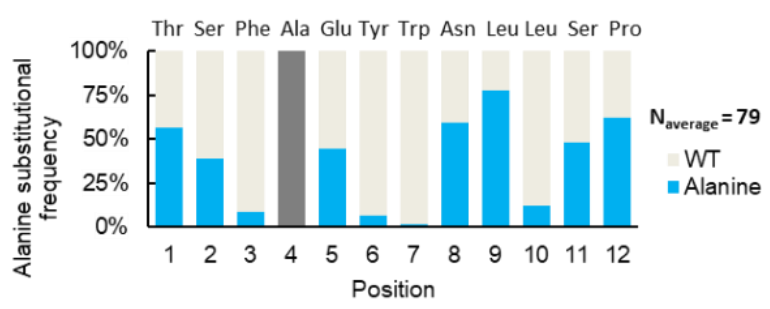

B) A subset of identified sequences

\begin{tabular}{ccccccccccccc}
\hline Entry & \multicolumn{11}{c}{ Example sequence } \\
\hline 1 & T & S & F & A & E & Y & W & N & $\underline{\mathbf{A}}$ & L & $\underline{\mathbf{A}}$ & P \\
2 & $\underline{\mathbf{A}}$ & S & F & A & E & Y & W & $\underline{\mathbf{A}}$ & $\underline{\mathbf{A}}$ & L & S & $\underline{\mathbf{A}}$ \\
3 & T & S & F & A & E & Y & W & $\underline{\mathbf{A}}$ & $\underline{\mathbf{A}}$ & L & $\underline{\mathbf{A}}$ & $\underline{\mathbf{A}}$ \\
4 & T & S & F & A & E & Y & W & N & $\underline{\mathbf{A}}$ & L & S & $\underline{\mathbf{A}}$ \\
5 & T & $\underline{\mathbf{A}}$ & F & A & E & Y & W & N & $\underline{\mathbf{A}}$ & L & S & $\underline{\mathbf{A}}$ \\
\hline
\end{tabular}

C) 2-D pairwise alanine substitution frequency matrix

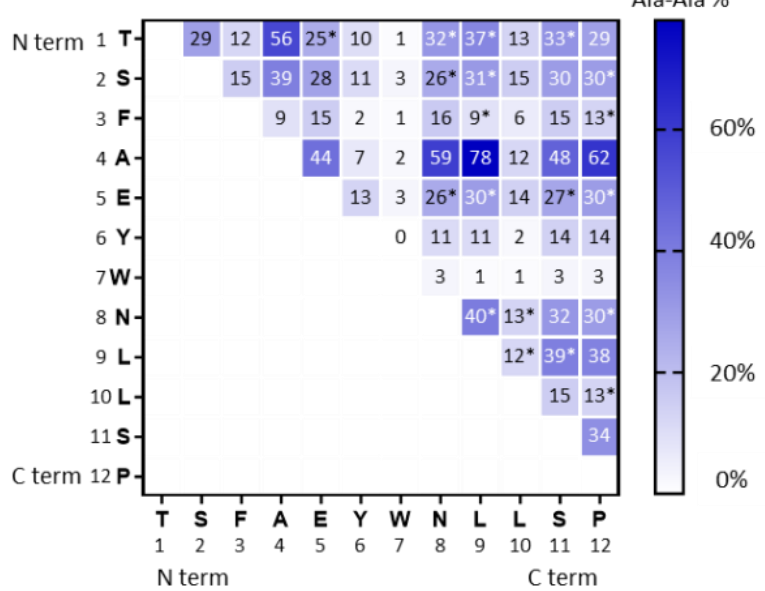

Figure 2. Combinatorial alanine scanning of peptide PMI combined with affinity selection mass spectrometry identifies novel multi-alanine substituted variants. A) The alanine tolerance was indicated by the single position alanine substitution frequency on the $y$-axis. Residues displaying low alanine substitution frequency (Phe3, Tyr6, Trp7 and Leu10) correspond to the hotspot residues. The $x$-axis indexes the PMI sequence from the $N$ - to the $C$-terminus. Ala4 is excluded. (B) A subset of identified sequences from the affinity selections. (C) The pairwise alanine tolerance is indicated by a substitution frequency matrix. Each box represents the pairwise alanine substitution frequency (Ala-Ala\%) of two residues, calculated as the ratio of number of observed simultaneous pairwise alanine substitutions to the total number of identified sequences, expressed as Ala-Ala $\%=$ $\left[\left(n_{\text {Ala,Ala }}\right) / n_{\text {total }}\right] \times 100 \%$. At $95 \%$ confidence level, twenty out of fifty-five total possible pairwise substitution frequencies are non-additive of single alanine frequencies. Nonadditive pairs are marked with asterisks $\left(^{*}\right)$. Ala4 is excluded.

\section{Pairwise SAR analysis reveals non-additive Ala-substituted pairs}

To identify pairs of non-additive Ala substitutions that might contribute to binding, a pairwise alanine substitution frequency matrix was generated ${ }^{9,14}$ (Figure 2C). The pairwise alanine substitution frequency (Ala-Ala\%) was computed by dividing the number of simultaneous pairwise alanine substitutions by the total number of identified sequences for each pair. Each box in the matrix presents a distinct pair of residues. For example, the residue pair (Thr1, Ser2) located in the first row and the second column of the matrix shows that $29 \%$ of the decoded sequences contained simultaneous (T1A, S2A) substitutions.

A statistical test showed that a number of Ala-Ala\%'s were not a mere product of two single Ala\%'s but were statistically different from simple combinations. A moderate nonadditive combination effect was revealed by comparing the observed pairwise alanine substitution frequency (Ala-Ala\%) observed to the product of single alanine frequencies (AlaAla\%)Additive. The theoretical additive double-mutant frequencies were computed from large sets $(1,000$ sets) of randomly-generated and independent alanine-substituted sequences, in which the randomization of each position is weighted by its positional alanine substitution frequency ${ }^{14}$. Each set contains hypothetical peptides sequences that follow the positional SAR. If a non-additive combination effect is present, (Ala- 
Ala\%)observed would not be equal to (Ala-Ala\%)Additive. To assess the statistical significance of the deviation, we compared the observed (Ala-Ala\%)observed to the theoretical (AlaAla\%)Additive. The difference (Ala-Ala\%)observed-(Ala-Ala\%)Additive was compared to the standard deviation $(\sigma)$ of the theoretical additive values calculated from the random sets of sequences and assessed by the $z$-test (Figure S2). To normalize for randomness, the statistical test was averaged by three replicate selections.

At $95 \%$ confidence (|z score $\mid>1.96 \sigma)$, nineteen out of fifty-five (34\%) pairwise Alanine substitution probabilities are statistically distinct from a simple combination of the corresponding two single alanine substitution probabilities (marked with asterisks in Figure 2C). Among these nineteen non-additive pairs, fourteen show negative cooperativity while five show positive cooperativity (Figure S2). Fourteen out of the nineteen pairs occur at non-hotspot residues, therefore, the non-additive combination effect is primarily observed elsewhere in the sequence.

\section{Pairwise SAR validation}

We envisioned the positions displaying high pairwise substitution frequencies in our combinatory alanine library (Figure 3A, extracted from Figure 2C, and Figure 3D) would tolerate double mutations. To validate the correlation between pairwise substitution frequencies and binding affinity of double-mutants, a series of $(i, i+4)$ pairwise alanine substituted peptides were prepared by automated fast-flow peptide synthesis ${ }^{28} \cdot(i, i+4)$ positions are chosen for subsequent macrocyclization ${ }^{29}$. The binding constant was determined by performing a competition assay with biolayer interferometry (BLI). Replacing the above average-frequency $(i, i+4)$ alanine-substituted pairs to alanine has minor impact on the binding affinity (Figure 3B; PMI, $K_{d}=7.7 \pm 4.5 \mathrm{nM}$ ).

Pairwise alanine-tolerated positions prompt sites amenable to further modifications, i.e., stapling. Side chain stapling is used for reinforcing helical structure and improving peptide stability toward proteases ${ }^{30-32}$. To leverage the pharmacological properties of the identified MDM2 binders, a hexafluorobenzene-mediated cysteine arylation reaction was employed to generate $(i, i+4)$ stapled PMI analogs (peptides 9-16) ) $^{33}$. BLI competition assays showed that the three highest frequency $(i, i+4)$ pairwise alanine substitutions (peptides 4, 5 and 8 in Figure $3 \mathrm{C}$ ) gave rise to the three highest affinity $(i, i+4)$ perfluoroaryl-stapled peptide binders at the corresponding positions. Peptides 12 and $\mathbf{1 3}$ showed slightly attenuated binding, and 16 exhibited a comparable binding to the parent PMI inhibitor $\left(K_{d}=7.7 \pm 4.5 \mathrm{nM}\right)$. This result indicates that peptides stapled at high $(i, i+4)$ alanine frequency positions can maintain low nanomolar binding affinity to MDM2. 


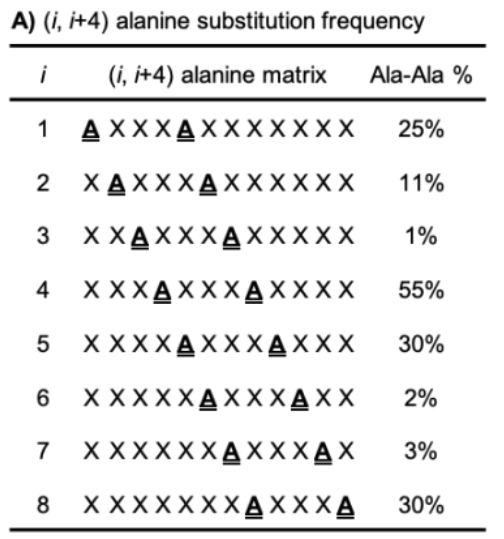

D) Peptide 1
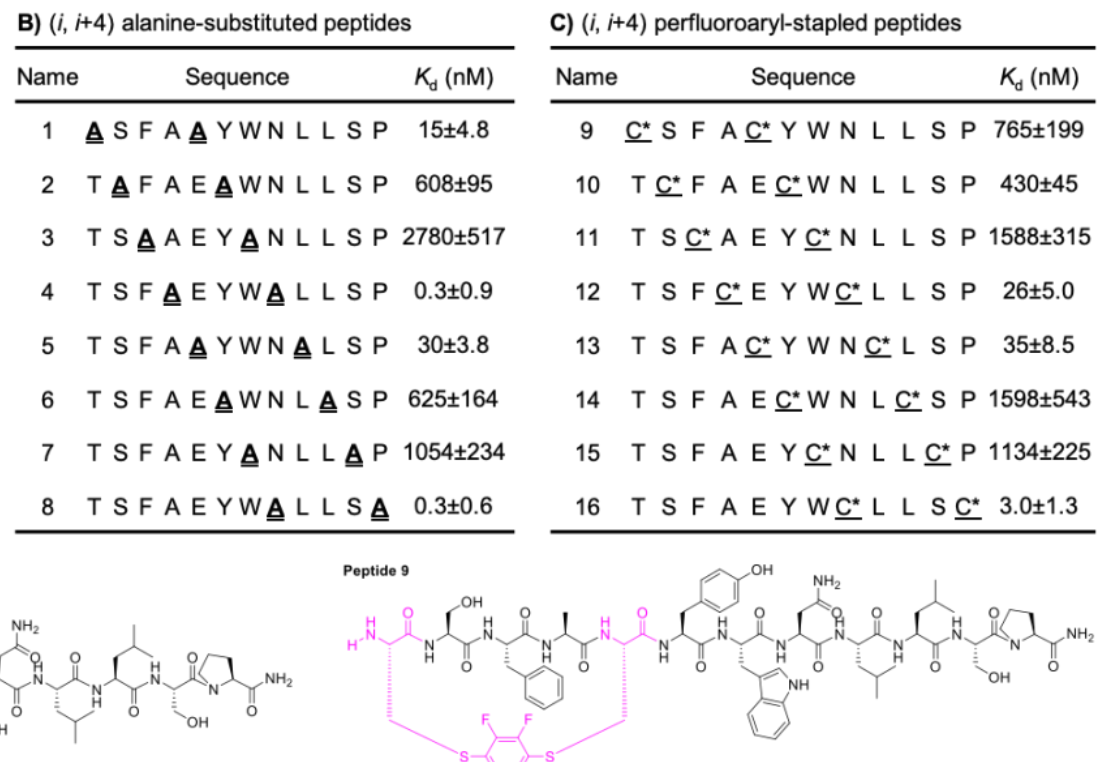

Figure 3. High-frequency alanine pairs observed in the affinity selection show high tolerance for double mutations. A) $(i, i+4)$ pairwise alanine substitution frequency extracted from Figure 2C. B) Binding affinity of $(i, i+4)$ pairwise alanine-substituted peptides. C) Binding affinity of $(i, i+4)$ perfluoroaryl stapled peptides. The three most potent stapled peptides correlate with the three highest frequency pairwise alanine substitutions. Therefore, the $(i, i+4)$ pairwise alanine substitution frequencies accurately indicate retention of high affinity in peptide binders substituted at the corresponding positions. Binding dissociation constants $\left(K_{\mathrm{d}}\right)$ were determined by a competition assay using BLI. D) Representative chemical structures of $(i, i+4)$ pairwise alanine-substituted peptide and $(i, i+4)$ perfluoroaryl stapled peptides. $\underline{\mathrm{C}^{*}}=$ cysteine stapled with hexafluorobenzene. $X=$ wild type residue or alanine. $\underline{\underline{A}}=$ alanine.

\section{Multiple alanine-substituted peptides retain potent binding affinity}

Raising the selection stringency, a small number of potent multiple alanine-substituted binders were identified (Figure 4A, see SI for selection condition). Using a smaller amount of the library increased the ligand-protein binding threshold, and consequently reduced the number of identified peptides. Although the seven non-hotspot residue substitutions can assemble a considerable number of unique combinations, only a fraction of these were observed in the identified sequences. Peptides with different alanine content were individually synthesized, and their binding affinity was validated by a competition assay using BLI. Several peptides exhibited low nanomolar binding affinity. Triple alanine sequences frequently occurred in the most stringent selection (Figure S3). They retained or even enhanced the protein-ligand binding interaction (Figure 4A).

The experimentally measured binding affinities of multi-alanine substituted PMI analogs generally do not correlate well with the theoretical binding affinities (Figure 4A). The

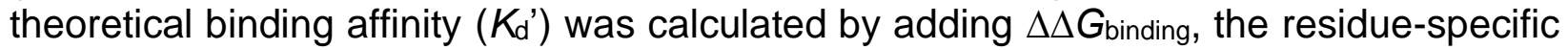
energy contribution of alanine mutations, of all substituted positions for a given peptide 
(Figure S4) and using the reported affinity of PMI $\left(K_{d}=3.2 \pm 1.1 \mathrm{nM}^{25}\right)$. Comparing the theoretical and measured binding affinities, peptides 17, 22 and 24 displayed ten-fold higher experimental $K_{d}$ values, while peptides 31, 32, 33 and $\mathbf{3 4}$ exhibited ten-fold lower experimental $K_{d}$ values relative to the calculated $K_{d}^{\prime}$ (Figure 4A). The theoretical $K_{d}$ ' given by simple addition of positional $\Delta \Delta G_{\text {binding }}$ can thus deviate significantly from the measured $K_{d}$, and generally does not accurately predict high-affinity multi-alanine substituted PMI variants.

Remarkably, peptide $\mathbf{3 4}$ has all of its non-hotspot residues substituted to alanine, while still maintaining a binding affinity comparable to PMI (Figure 4B). Single alanine mutagenesis predicts the $K_{\mathrm{d}}$ of peptide $\mathbf{3 4}$ to be 10 -fold weaker than PMI, while peptide 34, shows a $K d$ of $4.7 \pm 2.4 \mathrm{nM}$. Rigid molecular docking of peptide 34 to MDM2 using AutoDock Vina ${ }^{34}$ (except the side chains of the peptide, which were kept flexible) also shows that the calculated binding affinity was improved upon Ala substitutions at all seven non-hotspot residues, with a $\Delta \Delta G$ of $-3.4 \mathrm{kcal} / \mathrm{mol}$ for $\mathrm{PMI}$ and $-2.5 \mathrm{kcal} / \mathrm{mol}$ for PMI-Lys (Figure 4C, 4D and S5).

Even though it is generally accepted that multiple alanine substitutions are detrimental to binding affinity ${ }^{28}$, in our hands multi-alanine-substituted MDM2 binding peptides could be identified in the enriched sequences. A cooperative effect may take place when several alanine residues are introduced into the peptide. A modest propensity of alanine to stabilize helical structures may also account for the binding enhancement, as PMI forms an alpha-helix when co-crystallizing with $\mathrm{MDM}^{25}$. These multi-alanine-substituted peptides can provide templates for further structure modifications. 
A) Validation of multiple alanine-substituted peptides

\begin{tabular}{|c|c|c|c|c|}
\hline Name & Sequence & $\begin{array}{c}\text { Number of } \\
\text { alanine }^{*}\end{array}$ & $\begin{array}{l}\text { Theoretical } \\
K_{d}(\mathrm{nM})^{\star \star}\end{array}$ & $\begin{array}{c}\text { Measured } \\
K_{d}(\mathrm{nM})\end{array}$ \\
\hline PMI & TSFAEYWNLLSP & 0 & $3.2 \pm 1.1^{\star \star \star *}$ & $7.7 \pm 4.5$ \\
\hline 17 & TSFAEYW $\underline{\underline{A A} L S P K}$ & 2 & 0.5 & $32 \pm 4.2$ \\
\hline 18 & TS F A E Y W N $\underline{\underline{A} L}$ S $\underline{\underline{A}} K$ & 2 & 1.8 & $2.9 \pm 1.6$ \\
\hline 19 & TSFAE Y W N $\underline{\underline{\mathbf{A}}} L \underline{\underline{\mathrm{A}} P \mathrm{~K}}$ & 2 & 3.1 & $8.8 \pm 1.9$ \\
\hline 20 & TSFAEYWN $\underline{\underline{A} L} \underline{\underline{A A K K}}$ & 3 & 2.2 & $6.2 \pm 1.8$ \\
\hline 21 & 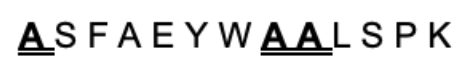 & 3 & 1.0 & $2.0 \pm 1.8$ \\
\hline 22 & 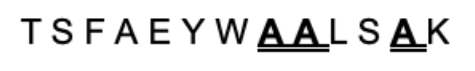 & 3 & 0.4 & $4.9 \pm 2.7$ \\
\hline 23 & T $\underline{\mathbf{A}} F \mathrm{~A} E \mathrm{Y} W \mathrm{~N} \underline{\underline{\mathbf{A}}} \mathrm{L} S \underline{\underline{\mathbf{A}}} \mathrm{K}$ & 3 & 15 & $3.7 \pm 1.5$ \\
\hline 24 & $\underline{\underline{\mathbf{A}} S} F A E Y W \underline{\underline{A} \mathbf{A}} L \underline{\underline{A}} P K$ & 4 & 1.2 & $59 \pm 11$ \\
\hline 25 & T S F A $\underline{\underline{\mathbf{A}} Y}$ W $\underline{\underline{\mathbf{A} A}} \mathrm{~L} S \underline{\underline{\mathbf{A}}} \mathrm{K}$ & 4 & 2.4 & $2.2 \pm 0.8$ \\
\hline 26 & T $\underline{\mathbf{A}} F \mathrm{~A} E \mathrm{Y} W \underline{\underline{\mathbf{A}} \mathbf{A} L S} \underline{\underline{\mathbf{A}} K} \mathrm{~K}$ & 4 & 3.0 & $1.0 \pm 1.0$ \\
\hline 27 & 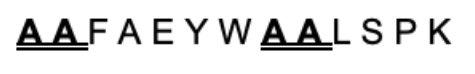 & 4 & 8.2 & $9.9 \pm 3.3$ \\
\hline 28 & $\underline{\underline{A} S}$ F A E Y W N $\underline{\underline{A} L} \underline{\underline{A A} K}$ & 4 & 4.1 & $4.7 \pm 1.5$ \\
\hline 29 & $\underline{\underline{\mathbf{A} S}}$ F A E Y W $\underline{\underline{\mathbf{A}} \mathbf{A} L} \underline{\underline{\mathbf{A} A K}}$ & 5 & 0.8 & $2.3 \pm 1.2$ \\
\hline 30 & $\underline{\underline{A}} S F A \underline{\underline{A}} Y W \underline{\underline{A} \mathbf{A} L S} \underline{\underline{A} K}$ & 5 & 4.6 & $1.9 \pm 1.9$ \\
\hline 31 & 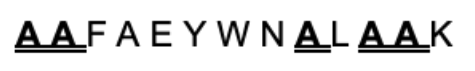 & 5 & 34 & $3.2 \pm 2.6$ \\
\hline 32 & 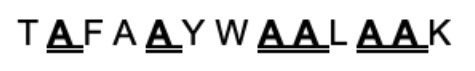 & 6 & 24 & $3.4 \pm 2.1$ \\
\hline 33 & 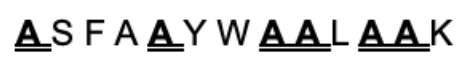 & 6 & 5.5 & $67 \pm 14$ \\
\hline 34 & 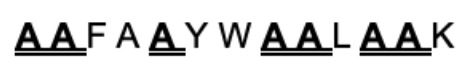 & 7 & 46 & $4.7 \pm 2.4$ \\
\hline
\end{tabular}

B) Peptide 34

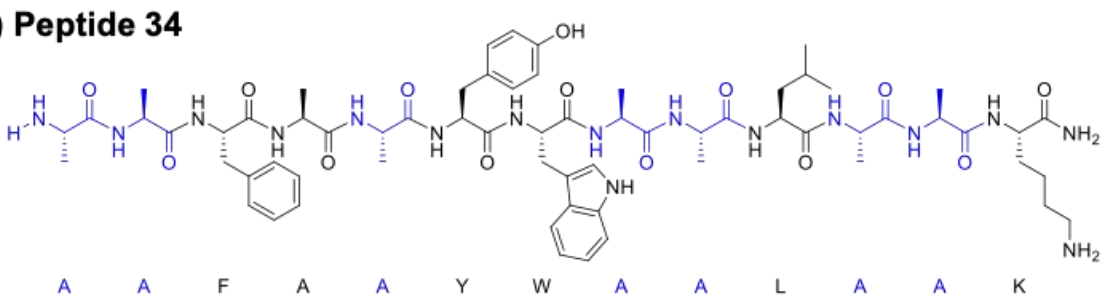

$\begin{array}{lll}\text { C) Molecular docking of PMI-K-MDM2 } & \text { D) Molecular docking of 34-MDM2 }\end{array}$

MDM2

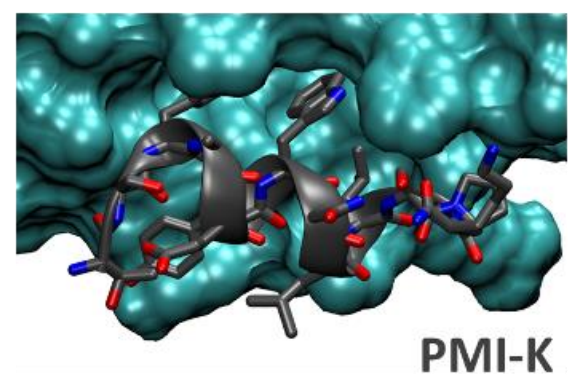

MDM2

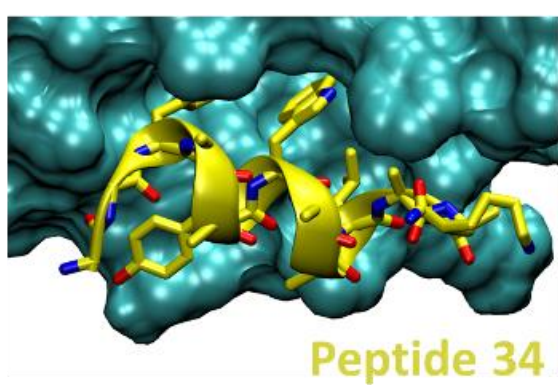


Figure 4. Multiple alanine-substituted peptides exhibit nanomolar binding affinity. By raising the selection stringency, alanine-substituted peptides were identified, and $\mathbf{A})$ a subset of the peptides were resynthesized and validated. Dissociation constants $\left(K_{d}\right)$ were determined by competition assay using BLI. *Ala4 is not considered. ${ }^{* *}$ Theoretical $K_{d}\left(K d^{\prime}\right)$ was calculated by adding the residue-specific energy contributions of individual alanine mutations (detailed calculation described in Figure S4). ${ }^{* *}$ The reported $K_{d}$ of $\mathrm{PMI}^{25}$. B) A 13-mer peptide 34 with seven alanine substitutions was identified $\left(K_{d}=\right.$ $4.7 \pm 2.5 \mathrm{nM}$ ). Molecular docking results of C) PMI-K (gray) and D) peptide 34 (yellow) bound to MDM2 (cyan).

\section{Multi-alanine substituted variants tolerate stapling}

To test the tolerance of multi-alanine-substituted peptides for subsequent modifications, we applied the $(i, i+4)$ perfluoroaryl-stapling strategy to alanine sites of the multi-alaninesubstituted peptides 22, 25, 27, 29 and 30 (Figure 5). Three stapling positions, (4, 8), (5, $9)$ and $(8,12)$, were chosen based on the tolerance for pairwise substitutions. The highest affinity $\left(K_{d}=0.7 \pm 0.5 \mathrm{nM}\right)$ was found with the $(8,12)$ perfluoroaryl-stapled peptide 40 . Except stapled peptide 35, stapled peptides 36, 37, 38 and 39 showed comparable binding affinity to PMI.

To assess the stability of stapled peptides, we performed a protease digestion assay using Cathepsin G (Figure S6A), which cleaves hydrophobic residues in serum ${ }^{35}$. Three stapled peptides were selected for assessment. Compared with the unmodified $\mathrm{PMI}-\mathrm{NH}_{2}$ peptide $\left(t_{1 / 2}=1.5 \mathrm{~min}\right)$, the half-life of stapled peptides $\mathbf{3 6}, \mathbf{3 8}$ and $\mathbf{3 9}$ increased to 11,20 and 23 mins respectively (Figure S6B). Noticeably, the 7-alanine-substituted peptide 34 $\left(t_{1 / 2}=20 \mathrm{~min}\right)$ also showed improved stability, but the 6 -alanine-substituted peptide $32\left(t_{1 / 2}\right.$ $=2.5 \mathrm{~min}$ ) did not. 


\begin{tabular}{|c|c|c|c|c|c|}
\hline Name & Sequence & $\begin{array}{l}\text { Stapling } \\
\text { sites }\end{array}$ & $\begin{array}{c}\text { Measured } \\
K_{\mathrm{d}}(\mathrm{nM})\end{array}$ & $\begin{array}{l}\text { Parent } \\
\text { peptide }\end{array}$ & $\begin{array}{l}\text { Parent } \\
\text { peptide }\end{array}$ \\
\hline 35 & $\underline{\mathbf{A}} \underline{\mathbf{A}} F \underline{\mathrm{C}^{*}} \mathrm{E} Y W \underline{\mathrm{C}^{*}} \underline{\mathbf{A}} \mathrm{L} S \mathrm{P}$ & $(4,8)$ & $316 \pm 27$ & 27 & $9.9 \pm 3.3$ \\
\hline 36 & $\underline{\mathbf{A}} S F \underline{C^{*}} E Y W \underline{C^{*}} \underline{\mathbf{A}} L \underline{\mathbf{A}} \underline{\boldsymbol{A}}$ & $(4,8)$ & $4.4 \pm 1.9$ & 29 & $2.3 \pm 1.2$ \\
\hline 37 & $T S F A \underline{C^{*}} Y W \underline{A} \underline{C^{*}} L S \underline{A}$ & $(5,9)$ & $1.6 \pm 1.3$ & 25 & $2.2 \pm 0.8$ \\
\hline 38 & $\underline{\mathbf{A}} S F A \underline{C^{*}} Y W \underline{\underline{A}} \underline{C^{*}} L S \underline{A}$ & $(5,9)$ & $1.5 \pm 1.1$ & 30 & $1.9 \pm 1.9$ \\
\hline 39 & $\underline{\mathbf{A}} S F A \in Y W \underline{C^{*}} \underline{A} L \underline{A} \underline{C^{*}}$ & $(8,12)$ & $3.3 \pm 2.1$ & 29 & $2.3 \pm 1.2$ \\
\hline 40 & $T S F A E Y W \underline{C^{*}} \underline{A} L S \underline{C^{*}}$ & $(8,12)$ & $0.7 \pm 0.5$ & 22 & $4.9 \pm 2.7$ \\
\hline
\end{tabular}

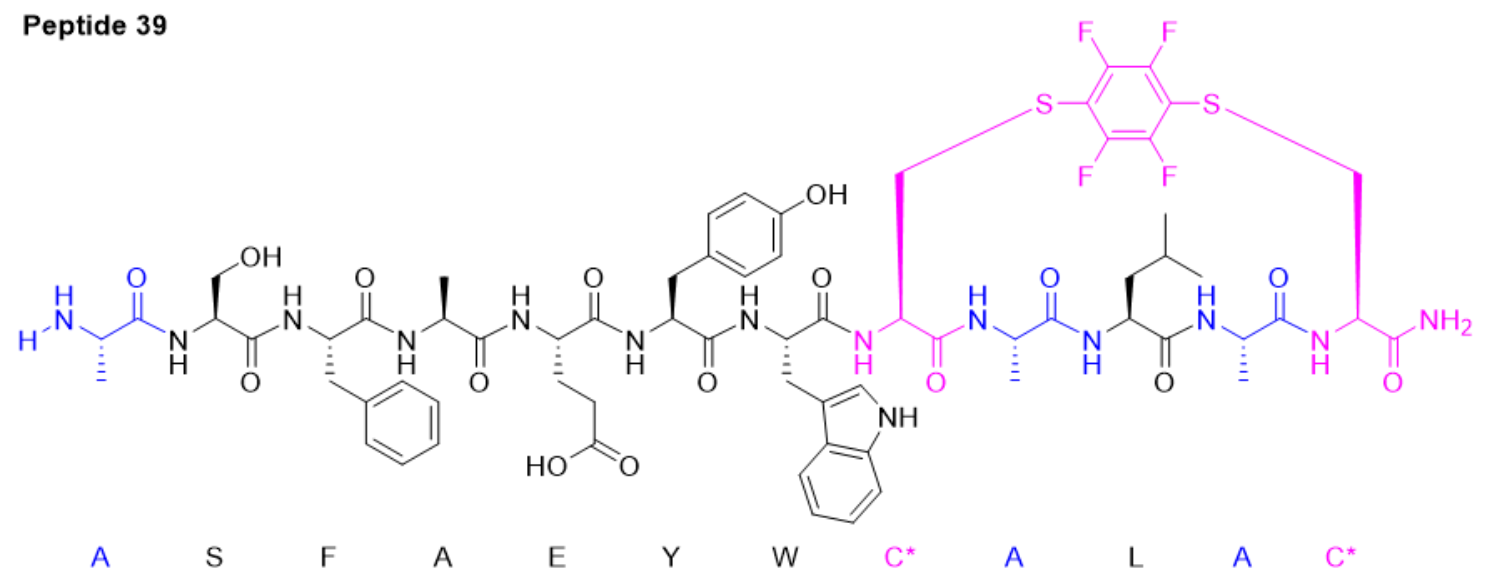

Figure 5. Perfluoroaryl-stapled and alanine-substituted peptides 35-40 with low nanomolar binding constant were generated at tolerable $(i, i+4)$ stapling sites (Figure $3 \mathbf{C}$ ) on potent PMI analogs 22, 25, 27, 29 and $30 . \underline{C^{*}}=$ cysteine stapled with hexafluorobenzene.

To expand this platform to other protein targets the combinatorial alanine scanning was used to discover peptide binders of antibody 12ca5 and the signaling protein 14-3-3 $\sigma$. The epitope used for $12 \mathrm{ca} 5$ has the sequence YPYDVPDYA; the previously characterized protein 14-3-3 $\sigma$ binder 14-3-3.6 was used for 14-3-3 $\sigma$ and its sequence can be found in Figure 6B. A beta-alanine spacer was used between the library construct and the Cterminal lysine. The combinatorial alanine scanning of YPYDVPDYA $\left(K_{d}=4 \mathrm{nM}\right)$ showed no alanine substitution at Asp4, Asp7 and Tyr8, which were previously reported as the hotspot residues of the epitope (Figure $6 \mathrm{~A})^{25}$. The combinatorial alanine scanning of peptide 14-3-3.6 $\left(K_{d}=3 \mathrm{nM}\right)$ showed no alanine replacement at phosphoserine 5 and nitro-phenylalanine 9 (Figure 6B), consistent with the close interactions these two residues have with $14-3-3 \sigma$, as seen in the co-crystal structure ${ }^{36}$. Remarkably, multiple alanine-substituted binders were identified under the most stringent condition as shown in the subset of sequences (Figure 6). 


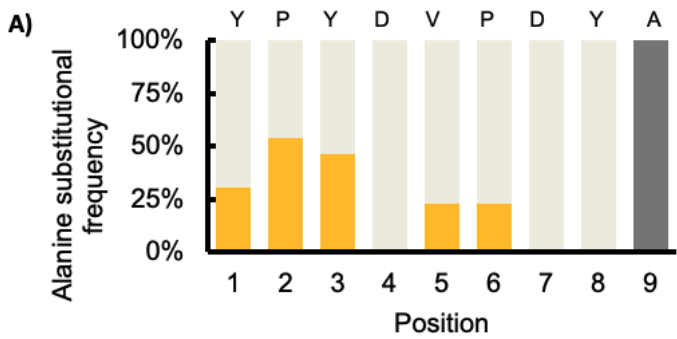

\begin{tabular}{|c|c|c|c|c|c|c|c|c|c|c|}
\hline \multirow[b]{3}{*}{$N=13$} & \multirow{2}{*}{$\frac{\text { Entry }}{1}$} & \multicolumn{9}{|c|}{ A subset of identified sequences } \\
\hline & & $\underline{\mathbf{A}}$ & $\underline{\mathbf{A}}$ & $Y$ & $\mathrm{D}$ & $\mathrm{V}$ & $\underline{\mathbf{A}}$ & $\mathrm{D}$ & $\mathrm{Y}$ & A \\
\hline & 2 & $\underline{\mathbf{A}}$ & $\underline{\mathbf{A}}$ & $\mathrm{Y}$ & $\mathrm{D}$ & $\underline{\mathbf{A}}$ & $\mathrm{P}$ & $\mathrm{D}$ & $\mathrm{Y}$ & A \\
\hline WT & 3 & $\underline{\mathbf{A}}$ & $P$ & $Y$ & D & V & $\underline{\mathbf{A}}$ & $\mathrm{D}$ & $\mathrm{Y}$ & A \\
\hline \multirow{2}{*}{ Alanine } & 4 & $\mathrm{Y}$ & $\underline{\mathbf{A}}$ & $\underline{\mathbf{A}}$ & $\mathrm{D}$ & V & $\mathrm{P}$ & $\mathrm{D}$ & $\mathrm{Y}$ & A \\
\hline & 5 & A & A & A & D & V & $\mathrm{P}$ & D & $Y$ & A \\
\hline
\end{tabular}

B)

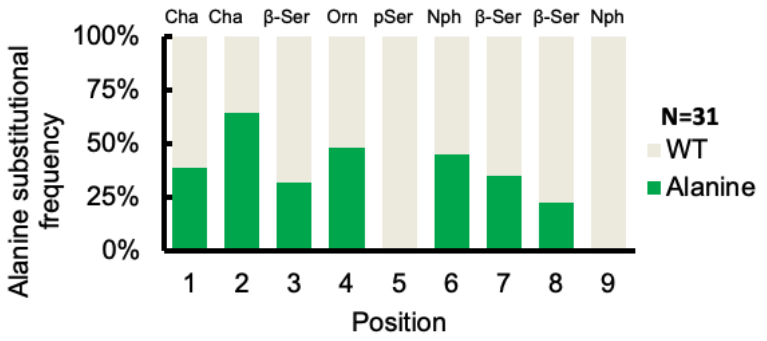

\begin{tabular}{cccccccccc}
\hline Entry & \multicolumn{10}{c}{ A subset of identified sequences } \\
\hline 1 & Cha & $\underline{\mathbf{A}}$ & $\beta$-Ser & Orn & pSer & Nph & $\beta$-Ser & $\underline{\underline{\mathbf{A}}}$ & Nph \\
2 & Cha & $\underline{\mathbf{A}}$ & $\beta$-Ser & $\underline{\mathbf{A}}$ & pSer & Nph & $\beta$-Ser & $\beta$-Ser & Nph \\
3 & Cha & Cha & $\beta$-Ser & Orn & pSer & $\underline{\mathbf{A}}$ & $\underline{\mathbf{A}}$ & $\beta$-Ser & Nph \\
4 & $\underline{\mathbf{A}}$ & $\underline{\mathbf{A}}$ & $\beta$-Ser & Orn & pSer & Nph & $\beta$-Ser & $\beta$-Ser & Nph \\
$\mathbf{5}$ & $\underline{\mathbf{A}}$ & $\underline{\underline{\mathbf{A}}}$ & $\underline{\underline{\mathbf{A}}}$ & $\underline{\underline{\mathbf{A}}}$ & pSer & Nph & $\beta$-Ser & $\underline{\underline{\mathbf{A}}}$ & Nph \\
\hline
\end{tabular}

Figure 6. Combinatorial alanine scanning is applicable to peptide binders for antibody

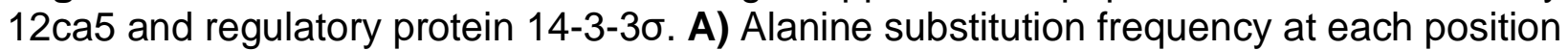
of the 12ca5 binder HA epitope (sequence: YPYDVPDYA). (B) Alanine substitution frequency at each position of the protein 14-3-3 $\sigma$ binder 14-3-3.6 construct (sequence: Cha, Cha, $\beta$-Ser, Orn, pSer, Nph, $\beta$-Ser, $\beta$-Ser, Nph); abbreviations: Cha, cyclohexyl alanine; $\beta$-Ser, beta-homoserine; Orn, ornithine; pSer, phosphoserine; Nph, 4-nitro phenylalanine. Wild-type alanine is excluded from the bar graph. 


\section{DISCUSSION}

We developed a label-free combinatorial alanine affinity selection platform to establish mutational tolerance, inform structure-activity relationships, and facilitate the optimization of peptide-based PPI modulators. Using various statistical analyses and peptide modifications, several sequence activity relationships were inferred. At the single substitution level, the determined alanine substitution frequencies differentiated between hotspot and non-hotspot residues of the MDM2 peptide binder PMI. At the double substitution level, statistical analyses of the pairwise alanine substitution frequencies identified a moderate but significant non-additive combination effect. In the pairwise alanine substitution frequency matrix, positions with high Ala-Ala\% tolerated double modifications. This matrix informed further modifications to retain affinity, validated by binding experiments with $(i, i+4)$ doubly-alanine-substituted peptides and $(i, i+4)$ stapled peptides.

Moreover, a handful of multiple alanine-substituted binders were found to maintain their binding affinity, thus revealing a new multi-alanine-substituted peptide binding modality. This discovery occurred when alanine was combinatorically introduced to peptide libraries that underwent affinity selections. The multi-substituted peptides can further tolerate cysteine-based stapling and still retain nanomolar binding affinity, despite the fact that stapling can potentially change the binding affinity of peptides ${ }^{29}$.

This study shows the potential of the combinatorial alanine scanning platform to identify multiple positions within a sequence that can simultaneously accommodate further modifications, for example, incorporating non-canonical amino acids. As a demonstration, we synthesized several perfluoroaryl-stapled and alanine-substituted peptide binders, which showed low nanomolar binding affinity to MDM2. The PMI-derived stapled peptides have increased protease resistance compared to the parent inhibitor. These high affinity stapled peptides may further be modified at the remaining alanine residues and provide a starting point in the development of the next generation of therapeutics targeting the oncogenic p53-MDM2 interaction.

Our study demonstrates a broad alanine tolerance landscape of peptide-based binders, provides insights into the cooperativity of combinatorial substitutions, and reveals a higher order binding modality with new multi-alanine-substituted sequences. This modality can guide peptide ligand optimization through chemical stapling and improve the throughput of hit-to-lead optimization processes. We envision that combinatorial alanine scanning can be a valuable tool for determining the feasible structural modifications of therapeutic peptide leads and enhancing their biophysical properties.

\section{Author Contributions}

$\dagger$ These authors contributed equally to this work.

\section{Notes}

B.L.P. is a co-founder of Amide Technologies and Resolute Bio. Both companies focus on the development of protein and peptide therapeutics. 


\section{Acknowledgements}

Financial support for this work was provided by Calico Life Sciences (to B.L.P.). Y.-C.L. gratefully acknowledges support from a Deutsche Forschungsgemeinschaft postdoctoral fellowship (DFG, LE 4224/1-1). We thank Bente A. Somsen and Christian Ottmann for generously providing the 14-3-3 protein, Anthony J. Quartararo for helpful discussions and suggestions, and Andrei Loas for valuable comments during manuscript preparation. We acknowledge the Biophysical Instrumentation Facility at MIT for providing access to the Octet Red96 Bio-Layer Interferometry System (NIH S10OD016326). Y.L., J.C.M., and Y.-S.L. thank the National Institute of General Medical Sciences (NIGMS) of the National Institutes of Health for financial support under award R01-GM124160 (to Y.-S.L.), the support of the Tufts IRACDA Program (NIGMS award K12-GM133314) for J.C.M, and the support of Laidlaw Undergraduate Research and Leadership Program at Tufts University for Y.L.

\section{REFERENCES}

(1) Nevola, L.; Giralt, E. Modulating Protein-Protein Interactions: The Potential of Peptides. Chem. Commun. 2015, 51 (16), 3302-3315.

(2) Cunningham, A. D.; Qvit, N.; Mochly-Rosen, D. Peptides and Peptidomimetics as Regulators of Protein-Protein Interactions. Curr. Opin. Struct. Biol. 2017, 44, 5966.

(3) Lee, A. C. L.; Harris, J. L.; Khanna, K. K.; Hong, J. H. A Comprehensive Review on Current Advances in Peptide Drug Development and Design. Int. J. Mol. Sci. 2019, 20 (10), 2383.

(4) Sohrabi, C.; Foster, A.; Tavassoli, A. Methods for Generating and Screening Libraries of Genetically Encoded Cyclic Peptides in Drug Discovery. Nat. Rev. Chem. 2020, 11 (8), 801-807.

(5) Derda, R.; Jafari, M. R. Synthetic Cross-Linking of Peptides: Molecular Linchpins for Peptide Cyclization. Protein Pept. Lett. 2018, 25 (12), 1051-1075.

(6) Bhat, A.; Roberts, L. R.; Dwyer, J. J. Lead Discovery and Optimization Strategies for Peptide Macrocycles. Eur. J. Med. Chem. 2015, 94, 471-479.

(7) Rogers, J. M.; Passioura, T.; Suga, H. Nonproteinogenic Deep Mutational Scanning of Linear and Cyclic Peptides. Proc. Natl. Acad. Sci. 2018, 115 (43), 10959-10964.

(8) London, N.; Movshovitz-Attias, D.; Schueler-Furman, O. The Structural Basis of Peptide-Protein Binding Strategies. Structure 2010, 18 (2), 188-199.

(9) Istomin, A. Y.; Gromiha, M. M.; Vorov, O. K.; Jacobs, D. J.; Livesay, D. R. New Insight into Long-Range Nonadditivity within Protein Double-Mutant Cycles. Proteins Struct. Funct. Genet. 2008, 70 (3), 915-924.

(10) Fowler, D. M.; Araya, C. L.; Fleishman, S. J.; Kellogg, E. H.; Stephany, J. J.; Baker, D.; Fields, S. High-Resolution Mapping of Protein Sequence-Function Relationships. Nat. Methods 2010, 7 (9), 741-746.

(11) Fowler, D. M.; Fields, S. Deep Mutational Scanning: A New Style of Protein 
Science. Nat. Methods 2014, 11 (8), 801-807.

(12) Leung, I.; Dekel, A.; Shifman, J. M.; Sidhu, S. S. Saturation Scanning of Ubiquitin Variants Reveals a Common Hot Spot for Binding to USP2 and USP21. Proc. Natl. Acad. Sci. U. S. A. 2016, 113 (31), 8705-8710.

(13) Li, X.; Lalic, J.; Baeza-Centurion, P.; Dhar, R.; Lehner, B. Changes in Gene Expression Predictably Shift and Switch Genetic Interactions. Nat. Commun. 2019, 10 (1), 3886.

(14) Gregoret, L. M.; Sauer, R. T. Additivity of Mutant Effects Assessed by Binomial Mutagenesis. Proc. Natl. Acad. Sci. U. S. A. 1993, 90 (9), 4246-4250.

(15) Lin, L.; Pinker, R. J.; Phillips, G. N.; Kallenbach, N. R. Stabilization of Myoglobin by Multiple Alanine Substitutions in Helical Positions. Protein Sci. 1994, 3 (9), 1430-1435.

(16) Kouadio, J. L. K.; Horn, J. R.; Pal, G.; Kossiakoff, A. A. Shotgun Alanine Scanning Shows That Growth Hormone Can Bind Productively to Its Receptor through a Drastically Minimized Interface. J. Biol. Chem. 2005, 280 (27), 25524-25532.

(17) Morrison, K. L.; Weiss, G. A. Combinatorial Alanine-Scanning. Curr. Opin. Chem. Biol. 2001, 5 (3), 302-307.

(18) Olson, C. A.; Wu, N. C.; Sun, R. A Comprehensive Biophysical Description of Pairwise Epistasis throughout an Entire Protein Domain. Curr. Biol. 2014, 24 (22), 2643-2651.

(19) Weiss, G. A.; Watanabe, C. K.; Zhong, A.; Goddard, A.; Sidhu, S. S. Rapid Mapping of Protein Functional Epitopes by Combinatorial Alanine Scanning. Proc. Natl. Acad. Sci. 2000, 97 (16), 8950-8954.

(20) Pál, G.; Fong, S.-Y.; Kossiakoff, A. A.; Sidhu, S. S. Alternative Views of Functional Protein Binding Epitopes Obtained by Combinatorial Shotgun Scanning Mutagenesis. Protein Sci. 2005, 14 (9), 2405-2413.

(21) Wells, J. A. Additivity of Mutational Effects in Proteins. Biochemistry 1990, 29 (37), 8509-8517.

(22) Touti, F.; Gates, Z. P.; Bandyopdhyay, A.; Lautrette, G.; Pentelute, B. L.; Bandyopadhyay, A.; Lautrette, G.; Pentelute, B. L. In-Solution Enrichment Identifies Peptide Inhibitors of Protein-Protein Interactions. Nat. Chem. Biol. 2019, 15 (4), 410-418.

(23) Vinogradov, A. A.; Gates, Z. P.; Zhang, C.; Quartararo, A. J.; Halloran, K. H.; Pentelute, B. L. Library Design-Facilitated High-Throughput Sequencing of Synthetic Peptide Libraries. ACS Comb. Sci. 2017, 19 (11), 694-701.

(24) Li, J.; Pazgier, M.; Lu, W.; Li, C.; Yuan, W.; Liu, M.; Zou, G.; Zella, D.; Tarasov, S. G.; Monbo, J. Structural Basis for High-Affinity Peptide Inhibition of P53 Interactions with MDM2 and MDMX. Proc. Natl. Acad. Sci. 2009, 106 (12), 46654670.

(25) Li, C.; Pazgier, M.; Li, C.; Yuan, W.; Liu, M.; Wei, G.; Lu, W. Y.; Lu, W. Systematic Mutational Analysis of Peptide Inhibition of the P53-MDM2/MDMX Interactions. J. 
Mol. Biol. 2010, 398 (2), 200-213.

(26) Hoe, K. K.; Verma, C. S.; Lane, D. P. Drugging the P53 Pathway: Understanding the Route to Clinical Efficacy. Nat. Rev. Drug Discov. 2014, 13 (3), 217-236.

(27) Chang, Y. S.; Graves, B.; Guerlavais, V.; Tovar, C.; Packman, K.; To, K.-H.; Olson, K. A.; Kesavan, K.; Gangurde, P.; Mukherjee, A.; Baker, T.; Darlak, K.; Elkin, C.; Filipovic, Z.; Qureshi, F. Z.; Cai, H.; Berry, P.; Feyfant, E.; Shi, X. E.; Horstick, J.; Annis, D. A.; Manning, A. M.; Fotouhi, N.; Nash, H.; Vassilev, L. T.; Sawyer, T. K. Stapled A-helical Peptide Drug Development: A Potent Dual Inhibitor of MDM2 and MDMX for P53-Dependent Cancer Therapy. Proc. Natl. Acad. Sci. 2013, 110 (36), E3445-E3454.

(28) Mijalis, A. J.; Thomas, D. A.; Simon, M. D.; Adamo, A.; Beaumont, R.; Jensen, K. F.; Pentelute, B. L. A Fully Automated Flow-Based Approach for Accelerated Peptide Synthesis. Nat. Chem. Biol. 2017, 13 (5), 464-466.

(29) Walensky, L. D.; Bird, G. H. Hydrocarbon-Stapled Peptides: Principles, Practice, and Progress. J. Med. Chem. 2014, 57 (15), 6275-6288.

(30) Li, X.; Tolbert, W. D.; Hu, H. G.; Gohain, N.; Zou, Y.; Niu, F.; He, W. X.; Yuan, W.; Su, J. C.; Pazgier, M.; Lu, W. Dithiocarbamate-Inspired Side Chain Stapling Chemistry for Peptide Drug Design. Chem. Sci. 2019, 10 (5), 1522-1530.

(31) Bernal, F.; Tyler, A. F.; Korsmeyer, S. J.; Walensky, L. D.; Verdine, G. L. Reactivation of the P53 Tumor Suppressor Pathway by a Stapled P53 Peptide. J. Am. Chem. Soc. 2007, 129 (9), 2456-2457.

(32) Azzarito, V.; Long, K.; Murphy, N. S.; Wilson, A. J. Inhibition of a-Helix-Mediated Protein-Protein Interactions Using Designed Molecules. Nat. Chem. 2013, 5 (3), 161-173.

(33) Spokoyny, A. M.; Zou, Y.; Ling, J. J.; Yu, H.; Lin, Y. S.; Pentelute, B. L. A Perfluoroaryl-Cysteine SNAr Chemistry Approach to Unprotected Peptide Stapling. J. Am. Chem. Soc. 2013, 135 (16), 5946-5949.

(34) Trott, O.; Olson, A. J. AutoDock Vina: Improving the Speed and Accuracy of Docking with a New Scoring Function, Efficient Optimization, and Multithreading. J. Comput. Chem. 2009, NA-NA.

(35) Zhan, C.; Zhao, L.; Wei, X.; Wu, X.; Chen, X.; Yuan, W.; Lu, W. Y.; Pazgier, M.; Lu, W. An Ultrahigh Affinity D-Peptide Antagonist of MDM2. J. Med. Chem. 2012, 55 (13), 6237-6241.

(36) Quartararo, A. J.; Gates, Z. P.; Somsen, B. A.; Hartrampf, N.; Ye, X.; Shimada, A.; Kajihara, Y.; Ottmann, C.; Pentelute, B. L. Ultra-Large Chemical Libraries for the Discovery of High-Affinity Peptide Binders. Nat. Commun. 2020, 11 (1), 3183. 


\section{TOC Figure}

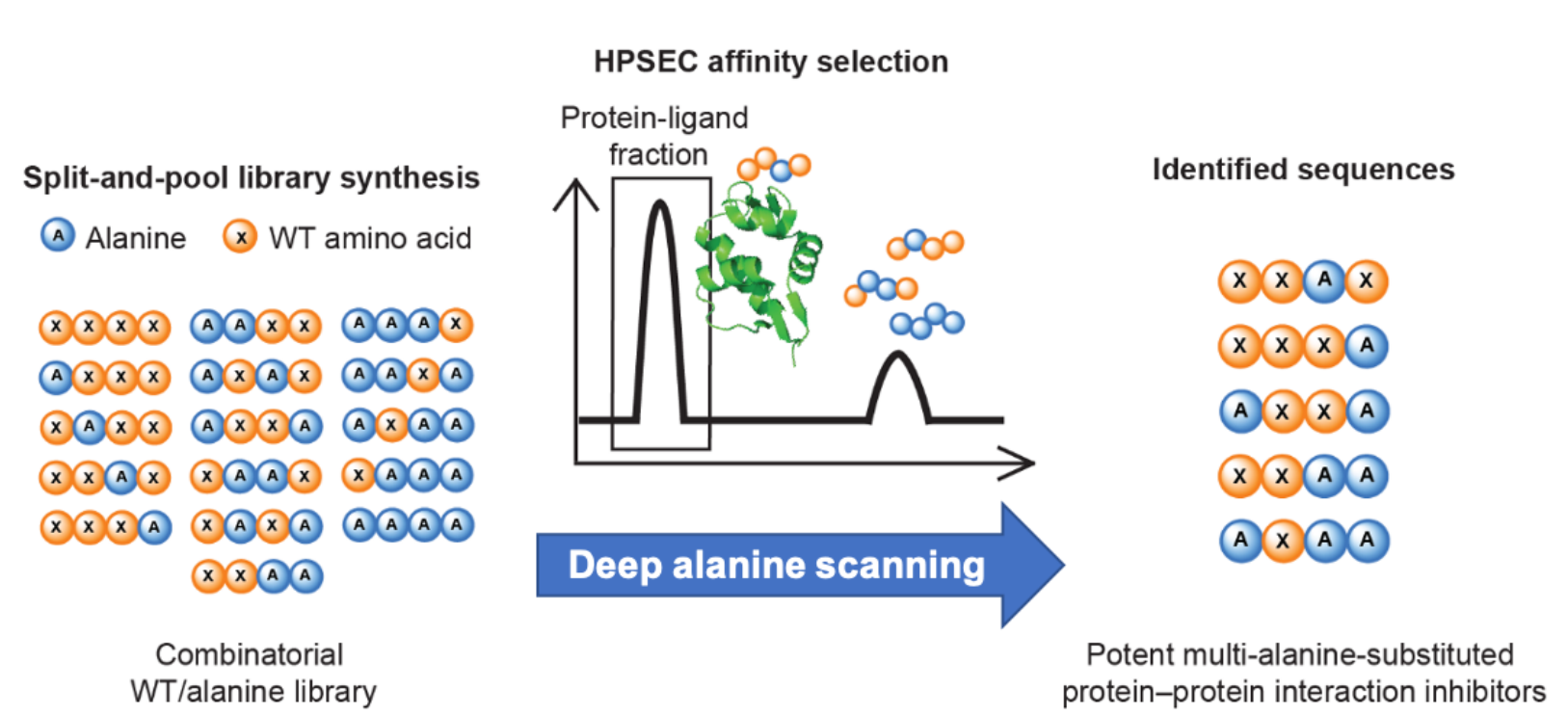

